\title{
A Patient-Adaptive Profiling Scheme for ECG Beat Classification
}

\author{
Miad Faezipour, Student Member, IEEE, Adnan Saeed, Suma Chandrika Bulusu, \\ Mehrdad Nourani, Senior Member, IEEE, Hlaing Minn, Senior Member, IEEE, \\ and Lakshman Tamil, Senior Member, IEEE
}

\begin{abstract}
Recent trends in clinical and telemedicine applications highly demand automation in electrocardiogram (ECG) signal processing and heart beat classification. A patient-adaptive cardiac profiling scheme using repetition-detection concept is proposed in this paper. We first employ an efficient wavelet-based beat-detection mechanism to extract precise fiducial ECG points. Then, we implement a novel local ECG beat classifier to profile each patient's normal cardiac behavior. ECG morphologies vary from person to person and even for each person, it can vary over time depending on the person's physical condition and/or environment. Having such profile is essential for various diagnosis (e.g., arrhythmia) purposes. One application of such profiling scheme is to automatically raise an early warning flag for the abnormal cardiac behavior of any individual. Our extensive experimental results on the MIT-BIH arrhythmia database show that our technique can detect the beats with $\mathbf{9 9 . 5 9 \%}$ accuracy and can identify abnormalities with a high classification accuracy of $97.42 \%$.
\end{abstract}

Index Terms-Beat classification, cardiac profile, electrocardiogram (ECG) signal processing, hash functions, packet processing, repetition, wavelet.

\section{INTRODUCTION}

\section{A. Background}

A UTOMATIC assessment of arrhythmia for patients has been a long time research. The cause of heart arrhythmias are due to the variations in the heart rate or irregularities and are characterized by the Electrocardiogram (ECG also known as EKG, abbreviated from the German Elektrokardiogramm) beats or patterns [1], [2]. The ECG signal is a representation of the bioelectrical activity of the heart representing the cyclical contractions and relaxations of the human heart muscles. To acquire the signal, ECG devices with varying number of electrodes (312) can be used. Multilead systems exceeding 12 and up to 120 electrodes are also available [3]. Accurate detection of the ECG beats is the key requirement for detecting all types of arrhythmia.

During the recording process, noise heavily affects the signal. In addition, the ECG signals collected from different people are heterogeneous, generally reflected by the variations in the

Manuscript received September 23, 2009; revised December 6, 2009 and April 26, 2010; accepted June 20, 2010. Date of publication June 28, 2010; date of current version September 3, 2010.

The authors are with the Quality of Life Technology Laboratory, The University of Texas at Dallas, Richardson, TX 75083 USA (e-mail: mxf042000@ utdallas.edu; axs055200@utdallas.edu; scb073000@utdallas.edu; nourani@ utdallas.edu; hlaing.minn@utdallas.edu; laxman@utdallas.edu).

Color versions of one or more of the figures in this paper are available online at http://ieeexplore.ieee.org.

Digital Object Identifier 10.1109/TITB.2010.2055575

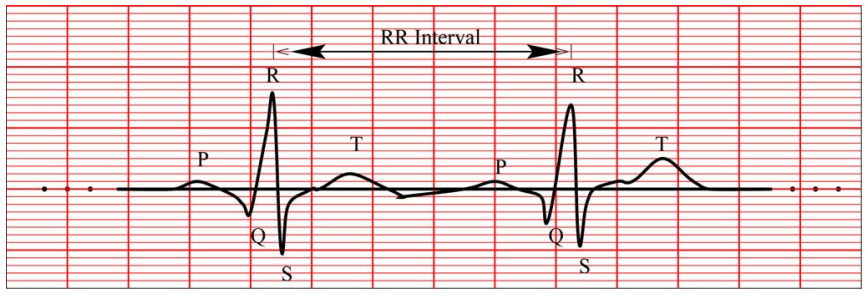

Fig. 1. Two pulses of a sample ECG waveform and the fiducial points P, Q, $\mathrm{R}, \mathrm{S}$, and T labeled.

amplitude of the beats. Hence, computationally intensive preprocessing is required for beat detection and feature extraction. The most important features include the information lying in the P, Q, R, S, and T waves of the ECG signal [1] (see Fig. 1). ECG beats should be classified based on these features in order to detect different types of arrhythmia.

In ambulatory ECG, all kinds of noise may occur simultaneously and unpredictably. Different kinds of noise include baseline wandering, electromyogram (EMG) noise, motion artifact, power-line interference, and electrode pop or contact noise [1], [4], [5]. ECG preprocessing generally takes care of denoising the ECG signal. The baseline wandering and the power-line interference (hum noise) are the most substantial noise and can strongly affect the ECG signal analysis. Baseline wandering (or trend) usually comes from respiration and lies between 0.15 and $0.3 \mathrm{~Hz}$. Other than these two noise elements, the remaining is wideband and usually complex stochastic processes, which also distort the ECG signal and affect the analysis. The power-line interference is a narrow-band noise centered at $60 \mathrm{~Hz}$ (or $50 \mathrm{~Hz}$ in Europe and some other countries) with a bandwidth of less than $1 \mathrm{~Hz}$ [4]. Usually, the ECG signal acquisition hardware can remove the power-line interference, but the baseline wandering and other wideband noise cannot be easily suppressed by hardware equipment without elaborate circuitry. However, software schemes can be used for ECG signal processing and suppression of these noise sources.

Several works have been done in the area of automatic ECG beat detection. However, many of them are not suitable when high accuracy is required. Many commercial tools are available for automatic beat detection, but their performance is not satisfactory. In our earlier work, we identified the possible enhancements in other previous algorithms with the help of Wavelet Transforms [6] to reduce the complexity and to increase the SNR of the ECG signal before detection [7]. To achieve this goal, we used LabVIEW graphical programming language. The 
software application could take the ECG signal, denoise it, and perform the beat detection efficiently. Possible enhancements include reducing the number of fiducial marks and reducing the number of thresholds that could, to a large extent, reduce the complexity.

After the preprocessing and beat detection, effective beat classification is required for correct analysis of different types of arrhythmia. The general trend is to develop automated systems to classify cardiac beats. This can significantly help simplify the diagnosis of heart diseases. For example, heart-rate variability or diagnosis of certain arrhythmia may take up to several hours when done by visual inspection. Even then, some vital information may be missed in between due to the tedious manual procedure. Therefore, computer-based beat classification is essential and becoming the norm in clinical applications [2].

So far, several techniques such as maximum likelihood, (artificial) neural networks [8], and support vector machines [9], [10] have been introduced for the ECG beat classification. These machine learning techniques map new data instances based on the information extracted from the annotated training data in the learning phase. Most techniques provide a global classifier that may not be always accurate for patient-specific cardiac variations. Automated arrhythmia-diagnosis systems that can provide high-classification accuracy rates for inter and intra-patient variation cases are still an active area of research.

\section{B. Main Contribution}

An efficient technique for classifying ECG beats is presented in this paper. A novel repetition-based detection technique has been adopted to classify, or more effectively, profile a patient's cardiac behavior. The key novelty of our approach is twofold. We first use an efficient method to accurately extract the QRS complexes of an ECG signal. Precise feature extraction is required for effective beat classification as well as other various electrocardiograph applications. We adopt a technique that uses wavelet analysis with adaptive thresholding for ECG preprocessing and feature extraction.

Second, we introduce a novel technique for profiling a patient's normal ECG. The main idea behind our approach is to consider ECG waveforms as data-packet streams and apply packet-processing techniques, namely, repetition-detection approaches [11], [12] to derive certain ECG patterns specific to a patient. Our approach is similar to the concept used in hardwarebased string matching and repetition-finding techniques used in worm detection in Internet networking [12]. Counter units and hashing functions are the concepts used for the processing. We derive an adaptive ECG profile for every patient, since ECG morphologies pretty much vary from person to person, as well as condition to condition for the same person. Our technique clearly identifies a normal region for a person and can, thus, identify abnormal beats that fall outside this normal region. Our technique is a local beat classifier that can be designed on top of a global classifier for performance enhancement. To the best of our knowledge, this type of ECG behavior profiling using packet-processing concepts (e.g., worm detection algorithm) has not been addressed in the literature. This paper has been integrated within a telemedicine server (PC) to enable remote high-speed cardiac processing and diagnosis [13].

\section{Paper Organization}

The rest of this paper is organized as follows. In Section II, we briefly review prior work related to ECG beat detection and classification. Our method for extracting most critical ECG fiduciary points is described in Section III. We propose our technique for classifying ECG beats in Section IV. Experimental results are summarized in Section $\mathrm{V}$ with a brief discussion on the key features of our classification technique. Finally, concluding remarks are in Section VI.

\section{PRIOR WORK}

\section{A. Denoising and Beat Detection}

Jiapu Pan and Willis J. Tompkins of the University of Wisconsin were perhaps the first to develop a real-time QRS detection algorithm on a Z-80 microprocessor [1].They performed denoising of the ECG signal using a bandpass filter which was built using cascaded high-pass and low-pass filters. Pan and Tompkins detected the fiducial points by finding the highest squared slope during high spectral energy of ECG waves. Based on our observations, this technique results in many more number of fiducial points than the actual QRS complexes. They considered two adaptive thresholds and chose the highest among the two thresholds extracted from the ECG signal and the integration of the ECG signal. A searchback algorithm was also applied if no QRS complex candidates were found within a certain time interval. They demonstrated a very good performance of $99.325 \%$ when tested against the MIT-BIH open-source arrhythmia database [14]. Note that, however, their technique required larger amount of processing as will be shown in Section III.

As for preprocessing of the ECG signal, noise cancellation requires different strategies for different noise sources. Thakor and Zhu [15] performed the noise reduction using an adaptive filter with constant or unity reference input, which was used to cancel baseline wander. However, this filter is not reliable for applications that require diagnostic ECG analysis.

Nonlinear filtering is a common approach to detect QRS complexes [1] in considerably less time and can be easily implemented. However, the main drawback of these algorithms is the frequency variation in QRS complexes, which adversely affects their performance. These methods can result in higher false positives and false negatives because the frequency band of QRS complexes generally overlaps with the frequency band of noise.

The authors in [6], [16], [17], and [18] employed the Wavelet Transform method for denoising of the ECG signal. In general, this technique decomposes the signal into various components that appear at different scales. It also uses a linear operation, which makes it suitable to preserve the important phase information of the signal. Dinh et al. [19] proposed to use the cubicspline wavelet and interpolation for accurate QRS detection. They concluded that wavelet functions that support symmetry and compactness achieve the highest accuracy on the ECG 
readings in MIT-BIH arrhythmia database. Though the wavelet transform approach does not discriminate between the noise and signal coefficients of the wavelet decomposition at low SNRs, it is still an attractive solution for nonstationary signals as it maintains the signal behavior.

\section{B. Beat Classification}

In addition to the automated ECG signal processing and beat detection, computer-based ECG beat classification is highly in demand as manual evaluation of the beats could be very time consuming. Methods such as pattern recognition, maximum likelihood, (artificial) neural network, and support vector machines have been widely used for classifying ECG beats [9], [10]. Machine-learning techniques learn from the samples of training data and map new data instances based on the information extracted from the annotated training data samples [8].

1) Support Vector Machines: Support vector machines (SVM) have been around for quite some time and have grabbed much attention for ECG beat classification [9], [10], [20]. In this technique, training datasets with known classes are given to the SVM program. Based on the training data, the SVM determines hyperplanes in the feature space for each class. The distances between the features of each instance and hyperplanes (classes) are computed and the hyperplane holding the minimum distance indicates that the instance is of that particular class. Each instance of data in SVM contains important features of the heart beat. For example, the instances of an SVM may contain QRS duration, RR interval, amplitude of $\mathrm{P}, \mathrm{Q}, \mathrm{R}, \mathrm{S}$, and $\mathrm{T}$ points. In other words, each instance is a vector of certain features of a beat, often called feature vector. The SVM methods provide good classification results using less training datasets and small feature vector sizes.

In beat classification using the SVM method, various ECG features may have information about different classes (types) of arrhythmia. Mohebbi and Moghadam [20] used the information of the ST segment in each beat of the ECG wave as elements of the feature vectors. Elevation of ST segment is proven to have information about myocardial ischemia episodes that may lead to certain heart attacks [20]. In another approach, transforms such as discrete Fourier transform, discrete cosine transform, discrete wavelet transform, and adaptive autoregressive coefficients are used in constructing the feature vector [21].

Ghongade and Ghatol [22] proposed using six different features in SVM classification method: mean R-peak value, mean power spectral density, area under QRS complex, energy of the signal, Q-S distance, and autocorrelation value. The authors claimed that this reduced feature set provides optimum results for characterizing four classes of heart beats [22].

Park et al. [23] first used higher-order statistics and Hermitebasis functions to extract ECG morphological features. The authors then used SVM to classify the beats into multiple classes. Their multiclass beat classification was done hierarchically, based on feature similarities between different classes.

2) Heuristic-Based Approaches: Apart from SVM, other classification techniques have been also studied. Christov et al. provided a thorough comparison of time-frequency ECG fea-

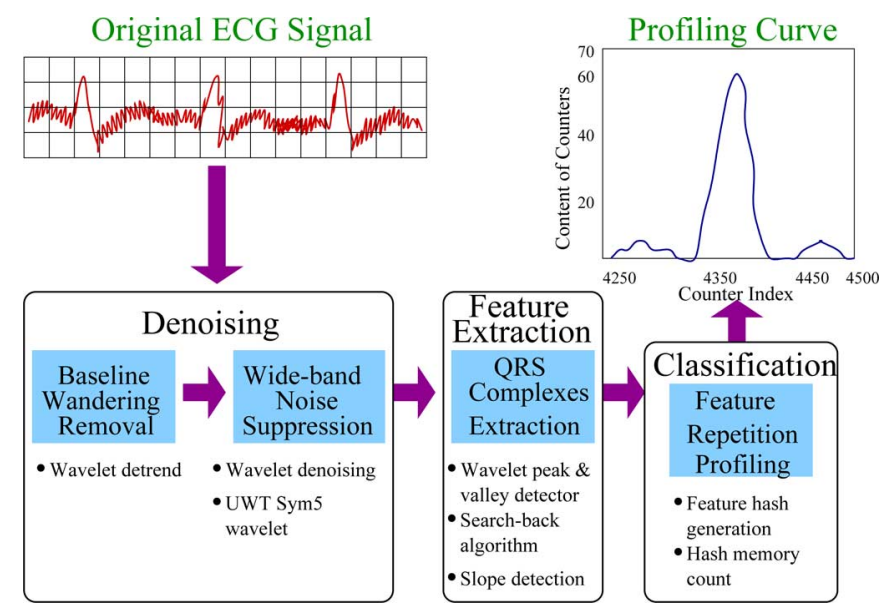

Fig. 2. Block diagram of our cardiac signal processing design.

tures for beat classification [24]. De Chazal et al. classified the beats by analyzing the RR intervals and ECG morphology features along with heart beat segmentation information [2]. They combined two linear-discriminant classifiers to make the final decision.

3) Artificial Neural Networks and Local Classifiers: Haseena et al. [25] use a hybrid of fuzzy clustering and artificial neural networks to discriminate between different classes of beats. The artificial neural networks along with the mixture of experts (MOE) approach introduced in [8] is a technique that uses a local classifier in addition to a global classifier in order to classify ECG beats. This technique is quite effective since a crisp global classifier that clearly defines a normal region for ECG features in a beat does not exist. Rather, a local classifier more or less particular to each individual is required to accurately classify the beats. Ubeyli [26] used a modified mixture of experts approach in which they gain a higher classification accuracy compared to the MOE and many artificial neural-network schemes. Pathological cardiac events identified using the method introduced in [27] is also a patient-adaptive classifier. This method analyzes the deviation of the RR interval from the mean value and the deviation of QRS patterns from the sustained rhythm. Our profiling scheme also falls in the same local-classifier category, which can be used on top of a global classifier.

\section{ECG-SIGNAL PROCESSING}

Denoising and detection of the QRS complexes in an ECG signal provide information about various cardiac abnormalities. It supplies evidence for the diagnosis of cardiac diseases. For this very important reason, it has earned a great respect in medical community. Unfortunately, the presence of noise and time-varying morphology makes the detection difficult. Fig. 2 shows the flow of our cardiac-signal processing.

\section{A. Preprocessing}

For ECG-signal processing, we use LabVIEW and related toolkits [28]. LabVIEW has wavelet analysis tools that are 
highly efficient for ECG denoising and feature extraction [29]. The wavelet type we used for discrete wavelet analysis is Symmlet 5 (sym5), which resembles ECG signal morphologies more than any other wavelet type. Moreover, Symmlet types highly support near-symmetry properties and provide high accuracy results on ECG signals [19]. Hence, sym 5 wavelet is by far a good and rationale selection.

The LabVIEW Advanced Signal Processing Toolkit (ASPT) provides the WA Detrend Virtual Instrument (VI) which can be used to remove the low-frequency baseline wandering (or trend) of a signal. In the WA Detrend VI, we also suggest using the sym5 wavelet as it resembles the QRS complex of ECG more than the other type of wavelets. An internal parameter called trend level is required for baseline removal [29]. The trend level is calculated as follows:

$$
\mathrm{LEVEL}_{\text {Trend }}=\left\lceil\frac{\log _{2} 2 t}{\log _{2} N}\right\rceil
$$

where $t$ is the observation duration and $N$ is the number of sampling points in observation time $t$. In this case, the highpass cutoff frequency is $0.8 \mathrm{~Hz}$ (below $1 \mathrm{~Hz}$ [5]).

After we suppress baseline wandering, the ECG signal becomes more stationary and explicit than the raw ECG signal. The remaining noise elements are complex stochastic processes with wideband nature, and cannot be efficiently suppressed or removed by the conventional digital-filtering approach [17]. To suppress the wideband noise, we chose to use the Wavelet Denoise VI module from the LabVIEW [29]. This module first decomposes the ECG signal into several subbands by applying the wavelet transform, and then modifies each wavelet coefficient by applying a threshold or shrinkage function, and finally reconstructs the denoised signal. In our design, we used undecimated wavelet transform (UWT) sym 5 with single level and soft thresholding for the wavelet denoising VI block setup. UWT results in the approximation wavelet coefficients and the detail coefficients at all decomposition levels. As UWT has the shiftinvariant property, it is highly efficient in robust-feature extraction and pattern-recognition applications. In addition, peak detection using UWT-based methods are more robust and less sensitive to noise, since UWT-based methods find zero-crossings in the multiscale UWT coefficients. These settings perform efficient denoising on the original ECG signal and smoothen the signal without suppressing ECG features such as the P and T waves. For effective feature extraction, we apply a wavelet denoise VI with UWT sym5 and multiple levels on the detrended signal to make only the QRS complexes of the signal more distinct.

\section{B. Feature Extraction}

The technique we deployed for ECG feature extraction is a hybrid approach of Pan and Tompkin's adaptive thresholding [1] combined with wavelet peak and valley detection [29], by which we achieved significant improvement compared to Pan and Tompkin's technique.

After detrending the signal and applying the wavelet denoising VI, the resulting signal would result in a zero DC offset.
TABLE I

COMPARISON OF OUR AND PAN-TOMPKIN's ALGORITHMS

\begin{tabular}{||c|c||}
\hline \hline Pan-Tomkin's Algorithm & Our Algorithm \\
\hline \hline $\begin{array}{c}\text { Digital Bandpass Filter } \\
\text { Denoising }\end{array}$ & $\begin{array}{c}\text { LabVIEW's Wavelet Detrending } \\
\text { and Denoising }\end{array}$ \\
\hline Integration waveform required & No Integrator required \\
\hline Two Threshold computations & Only one Threshold \\
\hline $\begin{array}{c}\text { PEAKI and NPKI computed } \\
\text { recursively in each window }\end{array}$ & $\begin{array}{c}\text { PEAK and NPK computed only } \\
\text { once by LabVIEW's WA Peak Detector }\end{array}$ \\
\hline Two $R R_{A v g}$ values required & Only one $R R_{A v g}$ required \\
\hline $\begin{array}{c}\text { Search back algorithm } \\
\text { for } 166 \% \text { of } R R_{A v g}, T H R / 2\end{array}$ & $\begin{array}{c}\text { Search back algorithm } \\
\text { for } 116 \% \text { of } R R_{A v g}, T H R / 8\end{array}$ \\
\hline \hline
\end{tabular}

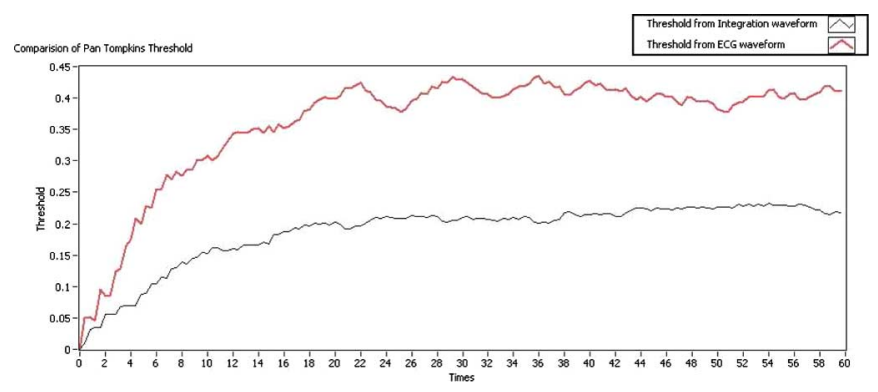

Fig. 3. Comparison of Pan-Tompkin's two thresholds.

We mark peaks that are above zero and valleys that are below zero by the help of WA Multiscale Peak/Valley Detection VI in LabVIEW. This particular VI uses the multiresolution wavelet analysis to detect peaks or valleys of a signal. By determining local maximums above a predefined threshold or local minimum below the threshold, the peaks or valleys can be extracted in this VI. Then, we apply an approach similar to Pan and Tompkin's method, but with some modifications (summarized in Table I), to dynamically set a threshold for beat detection. The peaks and valleys, detected from the peak/valley detector VI, now become candidates for signal peaks in Pan-Tompkin's algorithm [1].

In our implementation, only one set of threshold extracted from the denoised ECG can be applied, because the threshold calculated from integration waveform was found to be always lower than the other threshold, as seen in Fig. 3. Therefore, no integrator is required in our technique. This significantly lowers the amount of processing compared to Pan-Tompkin's.

Let us consider Peak as the array of peaks that LabVIEW peak-detector VI has found. The equations for adaptive thresholding are as follows:

$$
\left\{\begin{array}{l}
\text { PEAK }=\text { Maximum }(\text { Peak }) \\
\text { NPK }=\text { Minimum }(\text { Peak }) \\
\text { SPK }=0.125 \text { PEAK }+0.875 \text { SPK } \\
\text { THR }=\text { NPK }+0.25(\text { SPK }- \text { NPK) } .
\end{array}\right.
$$

A signal peak that is larger than the threshold THR is considered as a QRS complex, where the R point is detected. Each time a beat ( $R$ point) is found, we intentionally move the starting point of the next peak computation to a point that is $360 \mathrm{~ms}$ apart from the previous $\mathrm{R}$ point detected, as R-R intervals cannot physiologically be less than this timeframe [1].

Similar to Pan-Tomkins approach, a search-back algorithm is required if a beat is not found within a certain time interval. 
We maintain only one R-to-R average (instead of two) for the search-back algorithm, that being the average of the eight most recent R-R intervals found. If $n$ is the index of the current beat, $\mathrm{R}$-to-R average is computed as follows:

$$
\mathrm{RR}_{\mathrm{Avg}}= \begin{cases}\frac{1}{n-1} \sum_{i=0}^{n-2} \mathrm{RR}_{(n-i)}, & 2 \leq n \leq 7 \\ \frac{1}{8} \sum_{i=0}^{7} \operatorname{RR}_{(n-i)}, & n \geq 8 .\end{cases}
$$

If no beat has been detected within $116 \%$ of the current $\mathrm{R}$-to- $\mathrm{R}$ average, the search-back algorithm is applied. This is a percentage that has been found empirically [1]. In the searchback algorithm, we lower the threshold and start looking for a QRS complex from where the last $\mathrm{R}$ point is detected. Since our denoising technique only keeps the QRS complex of the signal and suppresses the $\mathrm{P}$ and $\mathrm{T}$ waves, a very low threshold can be used in this search-back stage. The new threshold we have applied in the search-back algorithm is as follows:

$$
\mathrm{THR}_{\text {new }}=\frac{\mathrm{THR}_{\mathrm{old}}}{8} .
$$

If a signal peak (SPK) exceeds this new threshold, we consider it as a beat ( $R$ point). The new SPK and threshold (THR) should then be updated accordingly.

\section{Fine Tuning}

A slope-detection technique has been integrated within our algorithm to rule out the possibility of misdetecting $\mathrm{T}$ waves as $\mathrm{R}$ beats. In some individuals (e.g., record 116 of the MITBIH arrhythmia database [14]), T waves are as high as (or even higher than) $\mathrm{R}$ waves. This may lead to misdetecting the beat if a peak-detection algorithm is used. In such cases, the slope is the key for beat detection. In general, $\mathrm{R}$ waves generate higher slopes compared to the $\mathrm{P}$ and $\mathrm{T}$ waves. Thus, when a peak is detected, the slope should be compared to the average slope of peaks, and removed from the list of beats if it is smaller than the average slope:

$$
\begin{gathered}
\text { Slope }_{\mathrm{Avg}}=\frac{\sum_{i=1}^{\# \text { of peaks }} y\left(\mathrm{Peak}_{i}\right)-y\left(\mathrm{Peak}_{i}-1\right)}{\# \text { of peaks }} . \\
\text { If }\left(\left(y\left(\operatorname{Peak}_{i}\right)-y\left(\operatorname{Peak}_{i}-1\right)\right)<\text { Slope }_{\mathrm{Avg}}\right), \text { then } \\
F=F-\left\{\text { Peak }_{i}\right\} .
\end{gathered}
$$

In the above formulations, $y\left(\operatorname{Peak}_{i}\right)$ and $y\left(\operatorname{Peak}_{i}-1\right)$ are the amplitudes of the $i$ th peak and one sample before the $i$ th peak, respectively, and $F$ refers to the set of peaks detected from the wavelet-peak detector in the LabVIEW.

To deal with the rare cases of records with inverted waves or records dissimilar to sym 5 wavelet, an effective approach is to square the entire signal. This would produce positive amplitudes for the $\mathrm{R}$ wave and would ensure that the squared $\mathrm{R}$ wave is relatively larger than $\mathrm{P}, \mathrm{Q}, \mathrm{S}$, and $\mathrm{T}$. Then, our beat-detection approach can be applied to the squared signal to find the beats. So, beforehand, a short timeframe (e.g., $10 \mathrm{~s}$ ) of each record should be analyzed to identify inverted wave records. After detrending and denoising the short record, absolute values can be given to our beat-detection approach. If the peaks were found at the locations where the original amplitude was negative, the signal can be declared as inverted, and the squared approach should be employed.

To find the Q and S points of the ECG waveform, we apply this underlying concept that a $\mathrm{Q}$ point is the closest valley right before $\mathrm{R}$ point, and an $\mathrm{S}$ point is the closest valley right after a detected $\mathrm{R}$ point. As mentioned earlier, the valley locations are found using the WA peak/valley detector with zero as the threshold. Since the signal has been detrended to zero-baseline wandering, this would locate all valleys that are below zero. When an R point is found, we search through the valley location array, and mark the maximum and minimum locations before and after that $\mathrm{R}$ point as $\mathrm{Q}$ and $\mathrm{S}$ points, respectively.

Table I summarizes the key differences between our design and Pan-Tompkins algorithm. Adaptive thresholding and employing the earlier search-back algorithm along with slope detection guarantee that our approach can take care of inter and intrapatient heart-rate variability or other dynamic variations over time due to change of the body position, breathing, electrode contact, etc., as reflected in the results of Section V.

\section{PRofiling ECG WAVEFoRM}

\section{A. Concept and Methodology}

The main idea in ECG profiling is based on the underlying concept of pattern matching used in Internet-packet processing. We treat ECG signals as data packets and apply similar methods used in packet processing such as identifying chunks of data packets that match certain (malicious) strings.

In the case of ECG packets, there is no global pattern (with fixed waveform dimensions) for a normal ECG waveform, making the problem more complicated. The reason is that ECG is a physiological phenomenon particular to each individual, which also depends on physical condition and environment of the person. Therefore, we need a patient-adaptive profiling scheme, similar to repetition-based pattern detection schemes, to derive a normal ECG pattern for an individual.

In general, string matching in networking applications is performed by comparing packet data bytes to a set of fixed signatures. In this process, data-packet chunks are analyzed to check whether any portion(s) of the packet stream matches those signatures. When predefined fixed signatures are not available for comparison, repetition-based pattern detection techniques can be used. The overall idea in repetition-based pattern detection is to identify strings that are frequently repeated at certain number of times [12]. In this technique, the input stream with total size of $|S|$ bytes is consistently broken into strings of $L$ bytes. The boundaries of these strings with distance $b$ are chosen consistently so that when a long string is repeated, the boundaries within that string are repeated at the same relative locations. To achieve this, the boundaries are decided based on the hash value of a certain sliding window of $W$-bytes. A boundary (border) is identified when the hash value is a fixed $l=\log _{2} L$ bit prime number. The data bits between two consecutive boundaries are examined for frequent occurrence. To achieve high-performance 


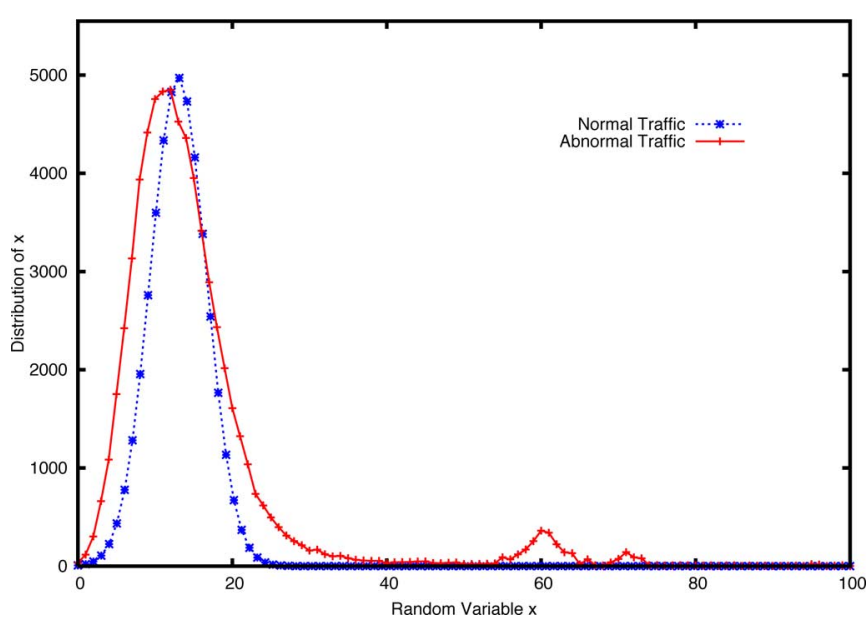

Fig. 4. Distribution of counter contents for $70 \mathrm{MB}$ of internet packets with abnormalities [12].

detection, without having to implement numerous counters, the shared counter technique is used. In this technique, multiple counters are used in which small storage units are designed to function in parallel. In the design, a multilevel hashing is used in two phases. The data bits between two consecutive boundaries are used to first generate a phase I hash. This hash value is then used in a second-phase hash to produce $m$ independent hash values that index into $m$ arrays. Assuming the width of each memory array to be $n$, each $m$ hash memory would have $2^{n}$ locations in depth. Each array location that is indexed, is incremented. Effectively, $m$ shared counters per signature during monitoring are used instead of one, adding to the accuracy of the conventional counting approach. If, say, $k$ out of $m$ counters exceed a certain threshold THR value, the signature is sent to other processing units as a worm suspect for further investigation. More accurate per signature counting is performed to confirm the suspicion afterwards.

In our prior work [12], we approximated the distribution of the number of counters with content $x$ to be a normal (bell-shaped curve) distribution:

$$
F_{X}(x)=\frac{1}{\sigma \sqrt{2 \pi}} e^{-\frac{(x-\mu)^{2}}{2 \sigma^{2}}}
$$

where $\mu=N P=|S| / 2^{n} L$ and $\sigma=\sqrt{\mu}$. In these formulations, the number of times our system enters phase II is $N=|S| / L$, and $P=1 / 2^{n}$ is the probability that phase I string would hash to one of the $2^{n}$ memory locations.

Based on the contents of the counters, a bell-shaped profiling curve would be provided, where anomalies would lie on the tail(s) of the curve [12].

In the design of our ECG waveform profiling system, similar concept as described earlier is used. We analyze the data in each heart beat and search for repetitions to extract an ECG pattern for any individual. Similar to our packet-processing technique where abnormalities are reflected as a long distorted tail with additional humps on the normal distribution curve scaled with a factor of the number of counters $2^{n}$ (see Fig. 4), abnormal ECG beats of the ECG profiling curve will be seen where humps exist on the tail of the bell-shaped curve.

\section{B. Algorithmic Fine Tuning}

Essentially, our behavioral analysis/profiling system is based on repetitions of some sort. The nature of these repeated parameters is application-specific and relevant to the features of each application. Thus, our system should be fine-tuned for each application to provide the profiling curve.

In our ECG profiling system, the data chunks chosen for frequent occurrence analysis is the time and amplitude information that lie in each QRS complex timeframe plus the time difference of the current and previous $\mathrm{R}$ points. To be consistent on the length of these data chunks, we chose a fixed timeframe of $150 \mathrm{~ms}$ centered at the R point, which empirically corresponds to the largest QRS complex [1]. Since multiple metrics are used here to quantify certain features of a heart beat, a (hash) function such as the summation of these quantities can be used to compact each instance (useful information between the borders of analysis) in a single string of data. The hash result would be the signature of phase 1 . In phase 2, multiple other (hash) functions can be applied to the phase 1 signature (e.g., mathematical functions such as mod, etc.) and multiple counters can be used to identify frequent occurrence of these hash results in parallel. We have chosen summation as our primary hash function, since as we will see further in the design, a linear function is used to maintain the range properties. Proper normalization is also required to result in an integer hash value. Essentially, when an $\mathrm{s}$ point is detected in the signal, the time and amplitude of all samples corresponding to $150 \mathrm{~ms}$ of the ECG signal centered at the $\mathrm{R}$ point, and the current RR distance are all summed up to form one string (hash value). This string is then further analyzed for frequent occurrence.

Similar to the second phase of the repetition-based patternmatching detection technique [12], each hash value is also an index to a presumable memory/counter location. That is, the counter that the hash value points to is incremented. However, since ECG is a physiological signal, each heart beat pattern would not exactly repeat with the same pattern as it had appeared in the previous beat(s). Therefore, hash values would never reproduce the exact same result, and thus, hash-memory contents would never be incremented if solely the aforementioned technique is used in our ECG profiling system. This is unlike Internet patterns where we search for repeated strings that exactly resemble one another in byte representation. To deal with this issue, we use a valid range where hash values would represent more or less a similar pattern, and increment counters that fall within that range. Pan and Tompkins defined an empirical range of $92-116 \%$ of the average of RR intervals, and claim that the beats that fall within this range indicate a normal range that do not require a search-back algorithm [1]. Based on this fact, we chose 25 , the difference between 92 and 116 as the number of memory locations we index and increment their contents. Essentially, for each hash generation, the content of 25 locations, 12 locations above, and 12 locations beneath the actual hash index would be incremented 


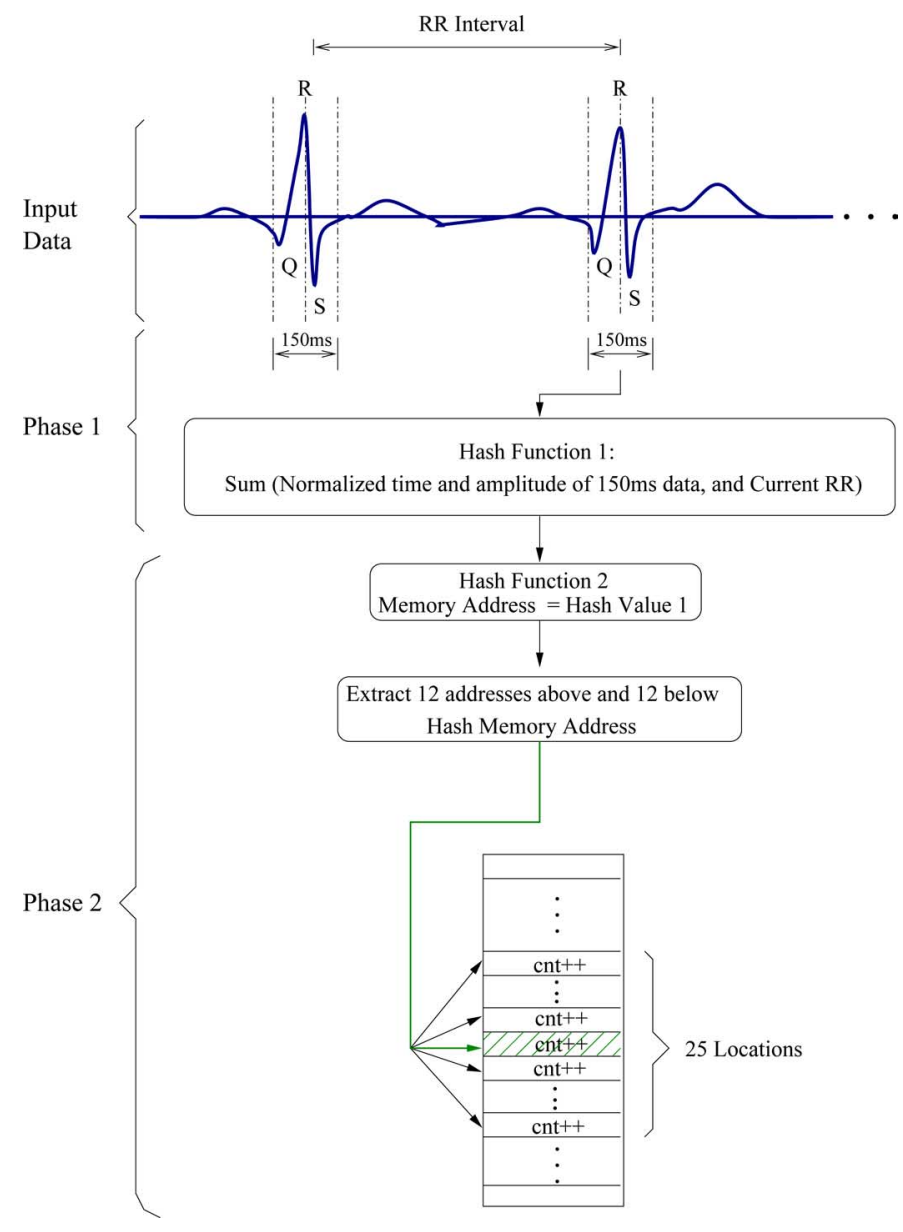

Fig. 5. Principles of our ECG profiling system.

Fig. 5 shows the principles of our methodology after feature extraction.

\section{Parametric Settings}

In our design, we have used one primary hash function (summation with normalization) and report the results. As discussed in repetition-based pattern detection techniques, multiple hash functions can be used in parallel to form the shared-counter approach. Other linear hash functions that can be interpreted into ranges properly can also be used. The shared-counter approach yields better results in terms of false positives (FP) and false negatives (FN) [12].

Our parametric settings for ECG profiling are summarized here. Note carefully that to show the true face of this application, we expressed all parameters in actual time measurements (second and millisecond). These metrics can be normalized to integer values by multiplying them with sampling frequency $\left(f_{s}=360\right.$ samples/s in our experiment).

1) $L \approx R R_{\text {interval }}$ : which is often in the range of $0.7-1.2 \mathrm{~s}$ representing the time gap expected between two consecutive heart beats.

2) $W=150 \mathrm{~ms}$ : the size of the sliding window around QRS region. Considering the fact that we use 2 bytes for amplitudes and time index, $W$ will be equivalent to $2 \times 0.150 \mathrm{~s} \cdot$ samples $/ \mathrm{s} \approx 108$ bytes.

3) $l=\log L=9$ : the size of the prime number for boundary check is 9 bits.

4) $b=R R_{\text {interval }}:$ which indicates that the borders (boundaries) of processing are one heart beat apart.

5) $m=1$ : showing only one memory unit in phase II.

6) $n=10$ : address bits of the memory arrays in phase II with $d \approx 16$ bits wide.

7) $k=1$ : indicating our policy in this particular experimentation to evaluate the system's performance using only one memory unit and one hash function (summation with normalized values) in the second phase.

8) THR: is the threshold value for counters. Since we chose the hash function to be the average content of counters for the best performance, the THR will be any value fallen out of normal range. In other words, the abnormal heart beats are those that do not fit in the bell-shaped normal behavior of counter values.

The features that are used in our profiling scheme provide classification results for different types of arrhythmia versus the normal ECG behavior. Repetition-detection analyses for features such as the $R-R$ interval and QRS duration provide classification results for the ventricular or atrial-premature beat classes. In other words, anomalies on the profiling curve indicate the presence of such premature beats. On the other hand, if the information lying in the ST segment of the signal is analyzed for frequent occurrence, abnormalities indicate the presence of myocardial ischemia episodes. Essentially, different ECG features or a combination of features, can collectively classify the beats into normal versus abnormal beats, where the abnormality type (class) depends on the ECG features used for profiling.

\section{EXPERIMENTAL RESULTS}

\section{A. Denoising and Feature-Extraction Simulation}

We have implemented our design in LabVIEW 8.5 graphical programming environment [28] and tested various ECG waveforms. Fig. 6 shows a raw ECG signal captured with a sampling frequency of $500 \mathrm{~Hz}$. After applying our denoising and beat detection technique, Fig. 7 shows the ECG signal that has been denoised and the $\mathrm{Q}, \mathrm{R}$, and $\mathrm{S}$ points marked for each beat.

\section{B. Performance Evaluation-Beat Detection}

Our algorithm, when evaluated against MIT-BIH arrhythmia database [14], achieved an overall beat detection accuracy of 99.59\% for the entire 30-min timeframe of the readings. Table II depicts the performance of our algorithm when implemented in LabVIEW.

False positives (FP) and false negatives (FN) have been reflected in the table as erroneously detected beats and missed beats, respectively. The overall error is calculated as follows:

$$
\text { Error }=\frac{\mathrm{FP}+\mathrm{FN}}{\text { Total \# of Beats }} .
$$

To determine the detection rate DER (accuracy), the true positive value TP (the number of correctly identified beats) is 


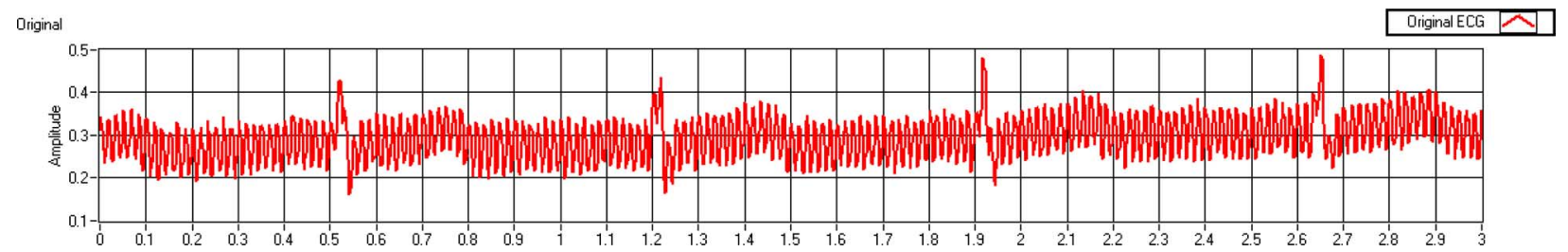

Fig. 6. Raw ECG signal.

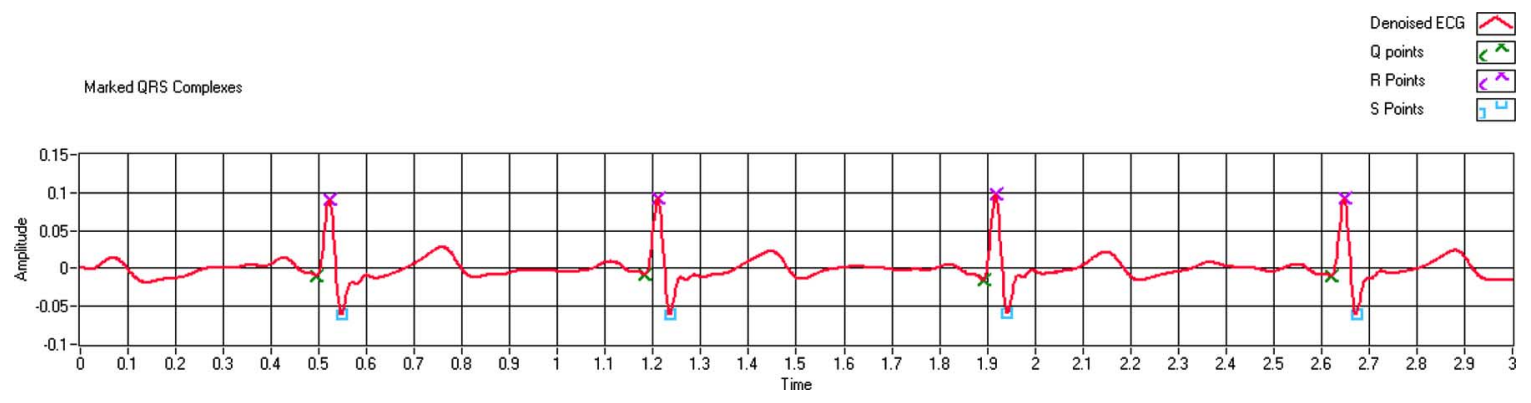

Fig. 7. ECG signal after wavelet denoising and QRS complex extraction.

used [31]:

$$
\text { DER }=\frac{\mathrm{TP}}{\text { Total \# of Beats }}
$$

Sensitivity (Se) and Specificity (Sp) are the most important parameters, used in literature, to assess the efficiency of any beat-detection algorithm [30]-[32]. They are defined as follows: ${ }^{1}$

$$
\begin{aligned}
& \mathrm{Se}=\frac{\mathrm{TP}}{\mathrm{TP}+\mathrm{FN}} \\
& \mathrm{Sp}=\frac{\mathrm{TP}}{\mathrm{TP}+\mathrm{FP}} .
\end{aligned}
$$

Our approach is able to detect beats with high accuracy even when considering inter and intrapatient variability. The key novelty is that it employs an adaptive thresholding scheme that dynamically adjusts the threshold for peak detection. In addition, a search-back algorithm is involved in the design that takes into consideration the history of the last eight previously detected beats, and lowers the threshold to search back for a beat that was not found in a nominal range using the previous threshold.

1) Handling Rare Cases: Our algorithm had originally performed poorly on those readings (e.g., datasets 108 and 200) that had inverted waves and did not look like the sym 5 wavelet [7]. To deal with the rare cases of records with inverted waves or records dissimilar to sym 5 wavelet, we have devised an effective heuristic by squaring entire signal and then applying our beat-detection approach to find the beats. This technique has been quite efficient, as we have achieved high beat-detection accuracy on records 108 and 200.

The performance of a few QRS detection algorithms that used digital filtering and wavelet analysis are compared in Table III. Our algorithm performs quite well just like other well-known

\footnotetext{
${ }^{1}$ Note that in some papers [8], [34], slightly different definitions were used. For example, positive prediction was defined as: $\mathrm{P}^{+}=\mathrm{TP} /(\mathrm{TP}+\mathrm{FP})$ and specificity was defined as: $\mathrm{Sp}=\mathrm{TN} /(\mathrm{TN}+\mathrm{FP})$.
}

approaches. Nevertheless, we achieved $99.62 \%$ accuracy for the first 23 readings (datasets 100-124 in Table II) that resemble normal ECG patterns, while Pan-Tomkins achieved 99.17\% accuracy for the same datasets [1].

\section{Performance Evaluation-Beat Classification}

We have applied our patient-adaptive profiling scheme on the entire 30-min timeframe of each record in the open-source MITBIH arrhythmia database [14]. In this experiment, we have used one hash function (summation with normalized values) in the first and second phases.

In general, abnormal ECG beats are reflected as distortions and humps on the tail of the bell-shaped curve. Highly distorted bell curve indicates the presence of too many irregular beats.

Fig. 8 shows the distribution of the counter contents for datasets 103,100, 200,112, 105, and 232, respectively, when considering a 1-min (first-minute) timeframe of profiling analysis. As can be seen, a bell-shaped curve represents the normal distribution of the content of the counters. The curve clearly depicts a normal region of the ECG beats. Any nonzero counter content that falls outside the normal region would indicate the existence of abnormal beats. Datasets 103 and 112 do not contain any abnormal beats, as the curves reflect this fact. Datasets 100 and 105 contain very few abnormal beats, which is reflected on the curves as very few counter contents outside the normal region. The abnormalities are of ventricular or atrial arrhythmia type. Datasets 200 and 232 contain too many abnormal beats, as a normal region can be hardly defined on the curves. All these results match the MIT-BIH arrhythmia database annotations for beat classification.

To numerically verify our results, we computed the percentage of area under the curve for the abnormal region and compared it with the percentage of abnormal beats within the same analysis timeframe of the MIT-BIH database. The area under 
TABLE II

PERFormance EVALUATION OF OUR QRS DETECTOR ON MIT-BIH ARRHYTHMIA DATABASE (30 MiN LONG EACH)

\begin{tabular}{||c|c|c|c|c|c||}
\hline \hline Data \# & \# of Beats & \# of FP & \# of FN & Error & Error\% \\
\hline 100 & 2265 & 0 & 0 & 0 & $0 \%$ \\
101 & 1861 & 4 & 3 & 7 & $0.37 \%$ \\
102 & 2180 & 0 & 0 & 0 & $0 \%$ \\
103 & 2078 & 0 & 0 & 0 & $0 \%$ \\
104 & 2222 & 5 & 5 & 10 & $0.45 \%$ \\
105 & 2565 & 0 & 0 & 0 & $0 \%$ \\
106 & 2021 & 0 & 0 & 0 & $0 \%$ \\
107 & 2128 & 7 & 10 & 17 & $0.79 \%$ \\
108 & 1755 & 0 & 2 & 2 & $0.11 \%$ \\
109 & 2521 & 9 & 6 & 15 & $0.59 \%$ \\
111 & 2130 & 12 & 0 & 12 & $0.56 \%$ \\
112 & 2532 & 4 & 3 & 7 & $0.27 \%$ \\
113 & 1789 & 0 & 0 & 0 & $0 \%$ \\
114 & 1897 & 27 & 2 & 29 & $1.52 \%$ \\
115 & 1946 & 0 & 0 & 0 & $0 \%$ \\
116 & 2387 & 6 & 23 & 29 & $1.21 \%$ \\
117 & 1530 & 4 & 4 & 8 & $0.52 \%$ \\
118 & 2284 & 13 & 0 & 13 & $0.56 \%$ \\
119 & 1981 & 0 & 0 & 0 & $0 \%$ \\
121 & 1856 & 2 & 2 & 4 & $0.21 \%$ \\
122 & 2468 & 0 & 0 & 0 & $0 \%$ \\
123 & 1514 & 1 & 0 & 1 & $0.06 \%$ \\
124 & 1613 & 12 & 12 & 24 & $1.48 \%$ \\
\hline 200 & 2595 & 2 & 0 & 2 & $0.07 \%$ \\
201 & 1961 & 4 & 2 & 6 & $0.3 \%$ \\
202 & 2127 & 2 & 3 & 5 & $0.23 \%$ \\
203 & 2967 & 0 & 6 & 6 & $0.2 \%$ \\
205 & 2648 & 1 & 1 & 2 & $0.07 \%$ \\
209 & 2998 & 3 & 2 & 5 & $0.16 \%$ \\
210 & 2641 & 3 & 4 & 7 & $0.26 \%$ \\
212 & 2740 & 2 & 2 & 4 & $0.14 \%$ \\
213 & 3215 & 0 & 26 & 26 & $0.8 \%$ \\
214 & 2252 & 5 & 7 & 12 & $0.53 \%$ \\
215 & 3352 & 9 & 10 & 19 & $0.56 \%$ \\
217 & 2210 & 11 & 3 & 14 & $0.63 \%$ \\
219 & 2147 & 0 & 0 & 0 & $0 \%$ \\
220 & 2041 & 0 & 0 & 0 & $0 \%$ \\
221 & 2428 & 10 & 2 & 12 & $0.49 \%$ \\
222 & 2481 & 27 & 20 & 47 & $1.89 \%$ \\
223 & 2596 & 16 & 17 & 33 & $1.27 \%$ \\
228 & 2043 & 0 & 4 & 4 & $0.19 \%$ \\
230 & 2248 & 1 & 1 & 2 & $0.08 \%$ \\
231 & 1569 & 4 & 0 & 4 & $0.25 \%$ \\
232 & 1776 & 2 & 2 & 4 & $0.22 \%$ \\
233 & 3060 & 15 & 24 & 39 & $1.27 \%$ \\
234 & 2745 & 0 & 0 & 0 & $0 \%$ \\
\hline \hline Total & Total \# & $\#$ of FP & $\#$ \\
Data & Beats) & (Beats) & (Beats) & Beats Not & Dverall|| \\
& $\mathbf{1 0 4 3 6 3}$ & $\mathbf{2 2 3}$ & $\mathbf{2 0 8}$ & $\mathbf{4 3 1}$ & $\mathbf{0 . 4 1 \%}$ \\
\hline \hline & & & & & \\
\hline
\end{tabular}

the curve for abnormal regions would be:

$$
A=\sum_{x=a}^{b} y(x)
$$

where $a$ and $b$ are the points (often on the tail of the curve) that fall out of the normal (bell-shaped) region, and $y(x)$ refers to the content of counter with index $x$. Table IV compares the performance of our scheme with the MIT-BIH arrhythmia database beat annotations (normal versus abnormal). The average of the results of the datasets is reported on row Avg, and the results for the entire $30 \mathrm{~min}$ of all 48 datasets are shown in the last row resulting in an overall classification accuracy of $97.42 \%$. The results of 30 one-min timeframes of analysis have been used for
TABLE III

COMPARISON OF BEAT DETECTION APPROACHES ON MIT-BIH ARRHYTHMIA DATABASE

\begin{tabular}{||c|c|c|c|c||}
\hline \hline Algorithm & Ref & $S e(\%)$ & $S p(\%)$ & $D E R(\%)$ \\
\hline \hline Pan Tompkins & {$[1]$} & 99.76 & 99.56 & 99.32 \\
\hline Dotsinsky et. al & {$[30]$} & 99.04 & 99.62 & Not specified \\
\hline Zhang et. al & {$[31]$} & 99.82 & 99.71 & 99.53 \\
\hline Zhou et. al & {$[32]$} & 99.43 & 98.55 & Not Specified \\
\hline Cvikl et. al & {$[33]$} & 99.82 & 99.83 & Not Specified \\
\hline Iliev et. al & {$[27]$} & 99.18 & 99.52 & Not Specified \\
\hline Proposed & - & $\mathbf{9 9 . 8 0}$ & $\mathbf{9 9 . 7 9}$ & $\mathbf{9 9 . 5 9}$ \\
\hline \hline
\end{tabular}

TABLE IV

Performance Evaluation of OUR ECG Profiling Scheme ON MIT-BIH ARRHYTHMIA DATABASE

\begin{tabular}{|c|c|c|c|}
\hline $\begin{array}{l}\text { Dataset } \\
(1-\mathrm{min})\end{array}$ & $\begin{array}{l}\text { MIT Abnormal } \\
\text { Beats }(\%)\end{array}$ & $\begin{array}{c}\text { Our Abnormal } \\
\text { Area }(\%)\end{array}$ & Error $(\%)$ \\
\hline 100 & 1.35 & 1.35 & 0 \\
\hline 101 & 0 & 0 & 0 \\
\hline 103 & 0 & 0 & 0 \\
\hline 104 & 100 & 97.5 & 2.5 \\
\hline 105 & 4.18 & 4.18 & 0 \\
\hline 109 & 100 & 97.62 & 2.38 \\
\hline 112 & 0 & 0 & 0 \\
\hline 115 & 0 & 0 & 0 \\
\hline 119 & 29.23 & 28.73 & 0.499 \\
\hline 121 & 0 & 0 & 0 \\
\hline 123 & 100 & 100 & 0 \\
\hline 124 & 0 & 0 & 0 \\
\hline 200 & 39.08 & 41.02 & 1.94 \\
\hline 203 & 9.8 & 10 & 0.2 \\
\hline 205 & 0 & 0 & 0 \\
\hline 212 & 100 & 100 & 0 \\
\hline 215 & 7.9 & 7.9 & 0 \\
\hline 217 & 100 & 99.2 & 0.8 \\
\hline 221 & 15.38 & 15.38 & 0 \\
\hline 223 & 1.25 & 1.25 & 0 \\
\hline 228 & 19.72 & 20.36 & 1.03 \\
\hline 231 & 100 & 100 & 0 \\
\hline 232 & 31.58 & 30.52 & 0.06 \\
\hline 234 & 0 & 0 & 0 \\
\hline Avg. & 31.64 & 31.45 & 0.18 \\
\hline $\begin{array}{c}\text { All (30-min) } \\
\text { of all } 48 \text { records }\end{array}$ & 68.53 & 65.95 & 2.58 \\
\hline
\end{tabular}

training. Then, the entire $30-\mathrm{min}$ profiling result was analyzed for each record.

Table $\mathrm{V}$ provides a performance comparison for a few published works. Our profiling scheme has achieved a comparable performance.

1) Training and Fine Tuning: Counter index in Fig. 8 is actually the hash result of the ECG features (RR interval, QRS duration, QRS amplitudes, etc.), which also points to a memory location. This is a value for quantifying ECG features. Each memory location is also a counter; i.e., whenever a hashed ECG feature value is produced, the content of that location is incremented by one. The $y$-axis corresponds to the content of memory with index $x$. For example, point $(4387,30)$ in Fig. 8(a) corresponds to the hash (memory) value of 4387 for the ECG features of particular beat. This memory location contains 30 beats. This figure does not show the time sequence of the beats. In other words, our approach does not demonstrate which beats are irregular in terms of time sequencing, rather it graphically 


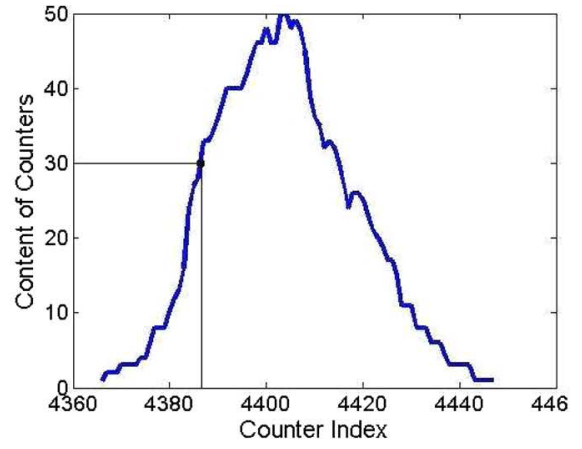

(a)

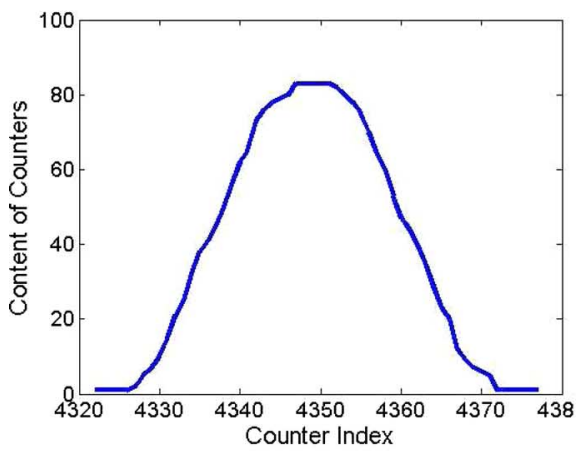

(d)

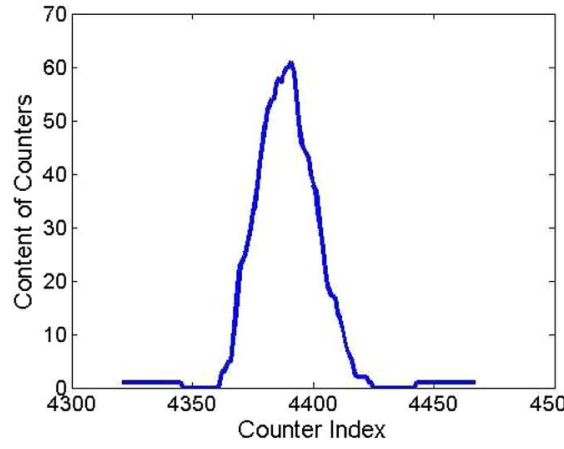

(b)

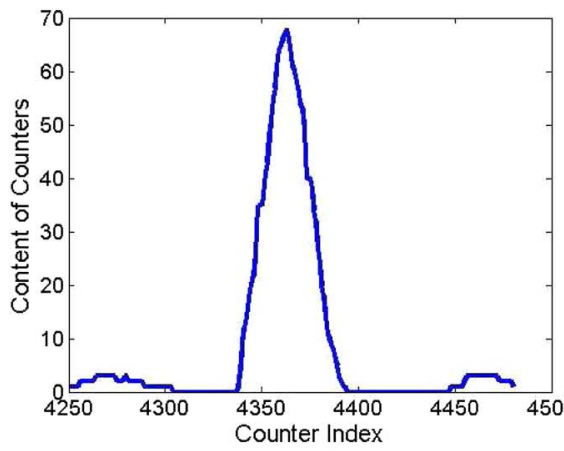

(e)

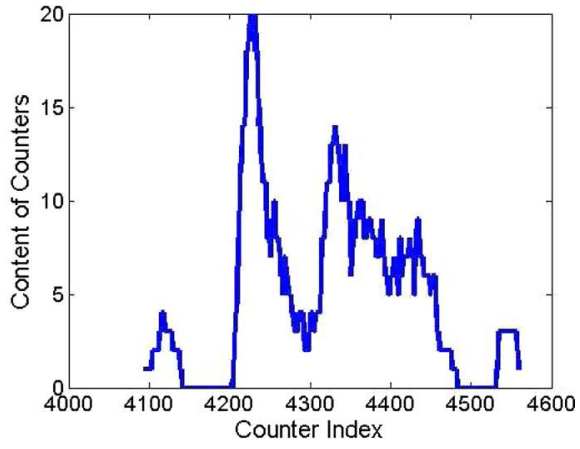

(c)

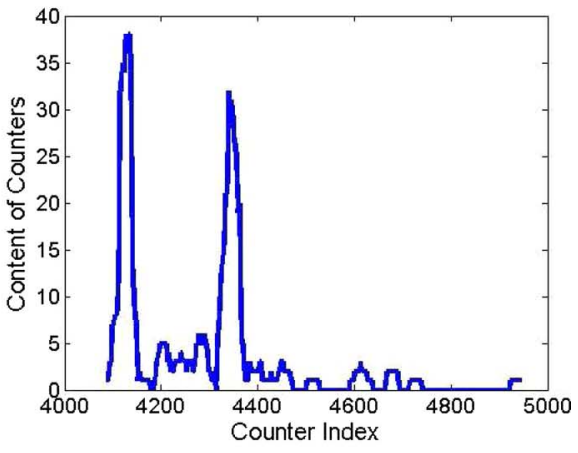

(f)

Fig. 8. Distribution of counter contents for MIT-BIH arrhythmia database readings. (a) Dataset 103. (b) Dataset 100. (c) Dataset 200. (d) Dataset 112. (e) Dataset 105. (f) Dataset 232.

TABLE V

COMPARISON OF BEAT ClASSIFICATION APPROACHES ON MIT-BIH ARRHYTHMIA DATABASE

\begin{tabular}{||c|c|c|c||}
\hline \hline Algorithm & Ref & Performance (\%) & \# of Features \\
\hline \hline de Chazal et. al & {$[2]$} & $S e=77.7, S p=98.8$ & 15 \\
\hline Christov et. al & {$[24]$} & $S e=94.77, S p=99.08$ & 15 \\
\hline Iliev et. al & {$[27]$} & $S e=95.7, S p=99.46$ & 3 \\
\hline Besrour et. al & {$[10]$} & $S e=98.38, S p=94.87$ & $10-15$ \\
\hline Haseena et. al & {$[25]$} & $D E R=97.54$ & 6 \\
\hline Proposed & - & $D E R=97.42$ & 1 \\
\hline \hline
\end{tabular}

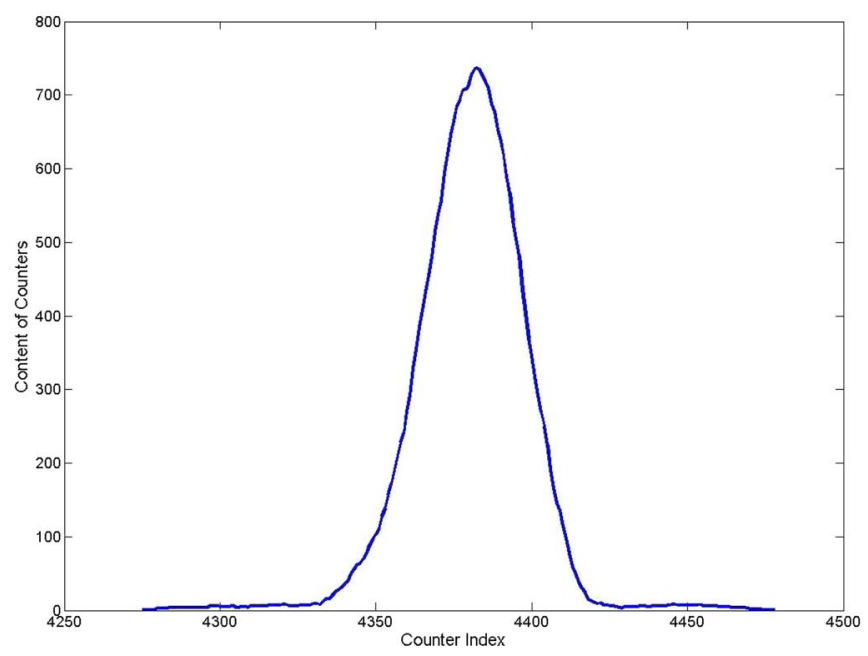

Fig. 9. ECG profiling curve for a 15-min timeframe of record 100 in MIT-BIH arrhythmia database.

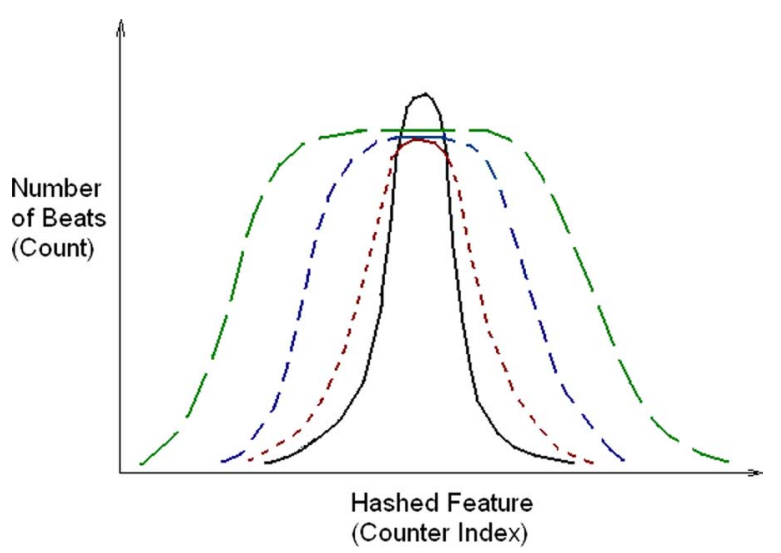

Fig. 10. Multiple profiling curves for different patients (or different physical conditions). Each condition has a specific normal range where the normal region of one condition may lie outside the normal region of another.

represents the presence of abnormal beats on the distorted tail of the curve. To pinpoint the irregular beats more accurately, our profiling scheme should be applied to shorter timeframes of analysis. In addition, longer runs of the profiling scheme may result in a huge normal (bell-shaped) area on the curve (see Fig. 9), making the abnormalities less visible. Moreover, just like other machine-learning/classification techniques, training our profiling system with samples of different arrhythmia types along with samples of normal beats allows our system to differentiate between different types of arrhythmia. The training 


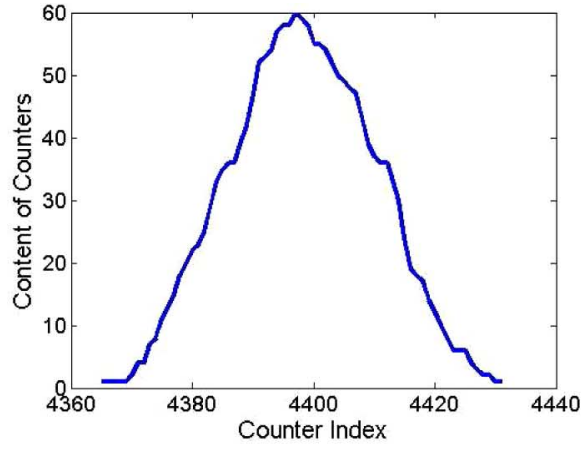

(a)

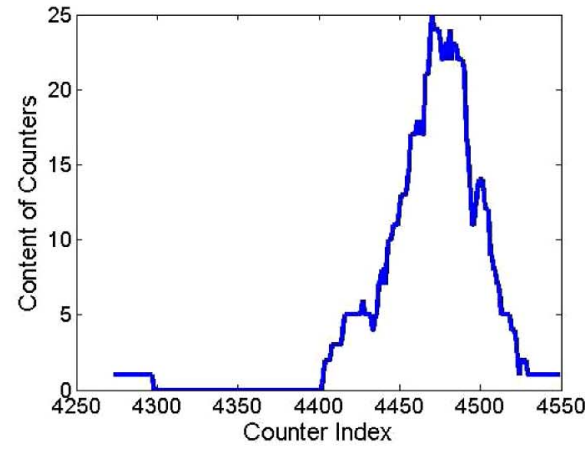

(b)

Fig. 11. ECG profiling curves for European ST-T database readings. (a) Dataset e0103. (b) Dataset e0105.

data must include a large number of mostly abnormal beats. Otherwise, if the system is trained with only abnormal beats, our system cannot identify the beats as abnormal, because the abnormal behavior would become the norm (pattern). Other local classification techniques follow the same rule. This is why a global classifier is still required to distinguish normal versus abnormal beats at a higher level. In this case, the abnormal area should be set to $100 \%$ instead of zero. Our profiling scheme can then be used as a local classifier, particular to any individual and zooming on short time intervals to classify the beats.

Note that different physical conditions of any individual can produce different profiling figures. In other words, the normal region for one condition may lie outside the normal region of another for the same patient (see Fig. 10). Our technique is an intelligent local classifier which is capable of identifying the presence of abnormal beats in an ECG profile, irrespective of the patient's physical condition.

2) Significance of the Proposed Method:

1) The significance of our study lies within the efficiency of the packetized data processing and the accuracy required in the ECG profiling technique. Since we consider the ECG record as a stream of packets, only one feature is sufficient for classification. This feature is a hash function applied to the samples to represent the information in only one string of data. The only information required from the ECG record is the $\mathrm{R}$ point. Our entire profiling technique is performed using this single feature. Note that feature extraction is a challenging and time-consuming task, especially for ECG signal where hardware (e.g., amplification and analog-to-digital conversion) and software (e.g., denoising, averaging, etc.) may require long processing time and may add to inaccuracy of data [10], [24]. Table $\mathrm{V}$ also compares the number of features required for a few recent techniques. Most approaches rely on extracting several features, adding to the complexity and overall latency of the beat classification procedure. These features generally include ECG morphology features, heart beat interval features $(\mathrm{P}, \mathrm{Q}, \mathrm{R}, \mathrm{S}$, and $\mathrm{T})$, frequency-based features, higher order cumulant features, hermite polynomials, time descriptors, wavelet coefficients, etc. Our technique relies more on the data stream corresponding to ECG beats than any particular feature. More specifically, our method has resulted in high accuracy in the range of other well-known approaches with only one feature.
TABLE VI

PERFORMANCE EVALUATION OF OUR ECG PROFILING SCHEME ON EUROPEAN ST-T DATABASE

\begin{tabular}{||c|c|c|c||}
\hline \hline Dataset & $\begin{array}{c}\text { European Abnormal } \\
\text { Beats (\%) }\end{array}$ & $\begin{array}{c}\text { Our Abnormal } \\
\text { Area (\%) }\end{array}$ & Error (\%) \\
\hline \hline $\mathrm{e} 0103$ & 0 & 0 & 0 \\
\hline $\mathrm{e} 0105$ & 1.72 & 1.69 & 0.03 \\
\hline \hline Avg. & 0.86 & 0.845 & 0.015 \\
\hline \hline $\begin{array}{c}30 \text { Records } \\
(\mathrm{e} 0103 \text {-e0154) }\end{array}$ & 43.94 & 38.71 & 5.23 \\
\hline \hline
\end{tabular}

2) Another significance of our study is that this type of packetized data processing could be effectively applied to other biometrics such as electroencephalogram (EEG) to detect the existence of abnormalities. The reason is that our type of repetition profiling is relatively feature-independent, and rather looks into the biometric signal as one single string of data. Hence, it can be employed and tuned by any irregularity/abnormality detection application.

3) Finally, as the third advantage of our approach, since our profiling architecture requires simple logic, e.g., counters, it could be easily implemented on customized hardware for clinical and/or wearable applications to raise an early warning flag for the existence of any irregularities.

In another experiment, we used the readings in the European ST-T database [35] to profile ST segment elevation abnormalities. This results in the myocardial ischemia arrhythmia types. The time and amplitude of 11 points lying $16 \mathrm{~ms}$ apart on the ST segment were extracted as the feature vector. The ST segment starts at the J point (which is the junction between the QRS complex and ST segment), and ends after a predefined interval of $160 \mathrm{~ms}$ [20]. According to [36], $\mathrm{J}$ is identified as a point in a sliding window of $20 \mathrm{~ms}$, which has a signal slope equal or less than $2.5 \mathrm{mV} \cdot \mathrm{s}^{-1}$ and is located within $20-120 \mathrm{~ms}$ after the $\mathrm{R}$ wave. The summation of these 11-point features has been used for the hashed string in both phases, which has then been analyzed for frequent occurrence. Fig. 11 shows the profiling curve for one-min timeframe of e0103 and e0105 readings in European ST-T database [35]. ST-segment elevations are reflected as the humps on the tail of the curve. Table VI shows the performance evaluation of our scheme compared to the European ST-T database beat annotations. The average of the 
results of the two datasets is reported on row Avg. Note that the last row in Tables IV and VI is not the sum of columns. Instead, those are the statistics for all 48 datasets in MIT-BIH and all first 30 records in European ST-T databases, respectively.

\section{CONCLUSION}

We introduced a technique for profiling the normal ECG waveform for any individual by first detecting the beats accurately. Our beat detection scheme was a hybrid of Pan and Tompkin's algorithm and wavelet analysis approach. We then classified the detected features of the beats by means of repetition-based packet-processing techniques. The outcome of our work is a profiling curve specific to any individual, representing a graphical view of the existence of abnormal beats. Our experimentation shows that a negligible classification error of $2.58 \%$ was observed when tested against the MIT-BIH arrhythmia database. This is a novel technique that can be used for automatic ECG beat classification in early warning monitoring systems.

\section{REFERENCES}

[1] J. Pan and W. J. Tompkins, "A real-time QRS detection algorithm," IEEE Trans. Biomed. Eng., vol. BME-32, no. 3, pp. 230-236, Mar. 1985.

[2] P. de Chazal, M. O'Dwyer, and R. B. Reilly, "Automatic classification of heartbeats using ECG morphology and heartbeat interval features," IEEE Trans. Biomed. Eng., vol. 51, no. 7, pp. 1196-1206, Jul. 2004.

[3] P. Zarychta, F. E. Smith, S. T. King, A. J. Haigh, A. Klinge, D. Zheng, S. Stevens, J. Allen, A. Okelarin, P. Langley, and A. Murray, "Body surface potential mapping for detection of myocardial infarct sites," in Proc. IEEE Comput. Cardiol., Sep./Oct. 2007, pp. 181-184.

[4] B.-U. Kohler, C. Henning, and R. Orglmeister, "The principles of software QRS detection," IEEE Eng. Med. Biol. Mag., vol. 21, no. 1, pp. 42-57, Jan./Feb. 2002.

[5] D. Zhang, "Wavelet approach for ECG baseline wander correction and noise reduction," in Proc. 27th IEEE Annu. Conf. Eng. Med. Biol., Sep. 2005, pp. 1212-1215.

[6] C. Li, C. Zheng, and C. F. Tai, "Detection of ECG characteristic points using wavelet transforms," IEEE Trans. Biomed. Eng., vol. 42, no. 1, pp. 21-28, Jan. 1995.

[7] M. Faezipour, T. M. Tiwari, A. Saeed, M. Nourani, and L. S. Tamil, "Wavelet-based denoising and beat detection of ECG signal," in Proc. IEEE-NIH Life Sci. Syst. Appl. Workshop, Apr. 2009, pp. 100-103.

[8] Y. H. Hu, S. Palreddy, and W. J. Tompkins, "A patient adaptive ECG beat classifier using a mixture of experts approach," IEEE Trans. Biomed. Eng., vol. 44, no. 9, pp. 891-900, Sep. 1997.

[9] M. H. Song, J. Lee, S. P. Cho, K. J. Lee, and S. K. Yoo, "Support vector machine based arrhythmia classification using reduced features," Int. J. Control, Autom., Syst., vol. 3, no. 4, pp. 571-579, Dec. 2005.

[10] R. Besrour, Z. Lachiri, and N. Ellouze, "ECG beat classifier using support vector machine," in Proc. 3rd IEEE Int. Conf. Inf. Commun. Technol.: From Theory Appl., Apr. 2008, pp. 1-5.

[11] B. Madhusudan and J. Lockwood, "Design of a system for real-time worm detection," in Proc. 12th Annu. IEEE Symp. High Perform. Interconnects, Aug. 2004, pp. 77-83.

[12] M. Faezipour, M. Nourani, and R. Panigrahy, "A real-time worm outbreak detection system using shared counters," in Proc. 15th Anпи. IEEE Symp. High Perform. Interconnects, Aug. 2007, pp. 65-72.

[13] A. Saeed, M. Faezipour, M. Nourani, S. Banerjee, G. Lee, G. Gupta, and L. S. Tamil, "A scalable wireless body area network for bio-telemetry," J. Inf. Process. Syst., vol. 5, no. 2, pp. 77-86, Jun. 2009.

[14] MIT-BIH Arrhythmia Database. (2010). [Online]. Available: www. physionet.org/physiobank/database/mitdb

[15] N. V. Thakor and Y. S. Zhu, "Applications of adaptive filtering to ECG analysis: Noise cancellation and arrhythmia detection," IEEE Trans. Biomed. Eng., vol. 38, no. 8, pp. 785-794, Aug. 1991.

[16] J. P. Martinez, S. Olmos, and P. Laguna, "Evaluation of a wavelet-based ECG waveform detector on the QT database," Comput. Cardiol., vol. 27, pp. 81-84, Sep. 2000.
[17] P. M. Agante and J. P. Marques de Sa, "ECG noise filtering using wavelets with soft-thresholding methods," Comput. Cardiol., vol. 26, pp. 535-538, Sep. 1999.

[18] J. S. Sahambi, S. N. Tandon, and R. K. P. Bhatt, "Using wavelet transforms for ECG characterization: An on-line digital signal processing system," IEEE Eng. Med. Biol. Mag., vol. 16, no. 1, pp. 77-83, Jan./Feb. 1997.

[19] H. A. N. Dinh, D. K. Kumar, N. D. Pah, and P. Burton, "Wavelets for QRS detection," in Proc. 23rd IEEE EMBS Int. Conf., Oct. 2001, pp. 18831887.

[20] M. Mohebbi and H. A. Moghadam, "An algorithm for automated detection of ischemic ECG beats using support vector machines," in Proc. 15th IEEE Conf. Signal Process. Commun. Appl., Jun. 2007, pp. 1-4.

[21] N. Acir, "Classification of ECG beats by using a fast least square support vector machines with a dynamic programming feature selection algorithm," Neural Comput. Appl. J., vol. 14, pp. 299-309, May 2005.

[22] R. Ghongade and A. Ghatol, "An effective feature set for ECG pattern classification," in Proc. ICMB, 2007, pp. 25-32.

[23] K. S. Park, B. H. Cho, D. H. Lee, S. H. Song, J. S. Lee, Y. J. Chee, I. Y. Kim, and and S. I. Kim, "Hierarchical support vector machine based heartbeat classification using higher order statistics and hermite basis function," Comput. Cardiol., pp. 229-232, Sep. 2008.

[24] I. Christov, G. Gomez-Herrero, V. Krasteva, I. Jekova, A. Gotchev, and K. Egiazarian, "Comparative study of morphological and time-frequency ECG descriptors for heartbeat classification," Med. Eng. Phys., vol. 28, no. 9, pp. 876-887, Nov. 2006

[25] H. H. Haseena, A. T. Mathew, and J. K. Paul, "Fuzzy clustered probabilistic and multi layered feed forward neural networks for electrocardiogram arrhythmia classification," J. Med. Syst., pp. 1-10, Aug. 2009.

[26] E. D. Ubeyli, "Eigenvector methods for automated detection of electrocardiographic changes in partial epileptic patients," IEEE Trans. Inf. Technol. Biomed., vol. 13, no. 4, pp. 478-485, Jul. 2009.

[27] I. Iliev, V. Krasteva, and S. Tabakov, "Real-time detection of pathological cardiac events in the electrocardiogram," Physiol. Meas., vol. 28, no. 3, pp. 259-276, 2007.

[28] National Instruments, User Manuals for LabVIEW Toolset Version 8.5.1, 2008

[29] LabVIEW for ECG Signal Processing. (2010). [Online]. Available: http://zone.ni.com/devzone/cda/tut/p/id/6349

[30] I. A. Dotsinsky and T. V. Stoyanov, "Ventricular beat detection in single channel electrocardiograms," BioMedical Engineering OnLine, vol. 3, p. 3, 2004.

[31] F. Zhang, J. Tan, and Y. Lian, "An effective QRS detection algorithm for wearable ECG in body area network," in Proc. IEEE Biomed. Circuits Syst. Conf., 2007, pp. 195-199.

[32] H.-Y. Zhou and K.-M. Hou, "Embedded real-time QRS detection algorithm for pervasive cardiac care system," in Proc. 9th IEEE Int. Conf. Signal Process., 2008, pp. 2150-2153.

[33] M. Cvikl and A. Zemva, "FPGA-oriented HW/SW implementation of ECG beat detection and classification algorithm," Digital Signal Process. vol. 20, no. 1, pp. 238-248, Jan. 2010.

[34] Z. Yong, H. Wenxue, X. Yonghong, and C. Jianxin, "ECG beats classification based on ensemble feature composed of independent components and QRS complex width," in Proc. IEEE Int. Conf. Comput. Sci. Softw. Eng., Dec. 2008, vol. 1, pp. 868-871.

[35] European ST-T Database. (2010). [Online]. Available: www.physionet. org/cgi-bin/ATM

[36] I. K. Daskalov, I. A. Dotsinsky, and I. I. Christov, "Developments in ECG acquisition, preprocessing, parameter measurement, and recording," IEEE Eng. Med. Biol. Mag., vol. 17, no. 2, pp. 50-58, Mar./Apr. 1998.

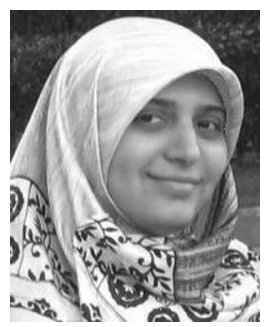

Miad Faezipour (S'06) received the B.S. degree in electrical engineering from the University of Tehran, Tehran, Iran, in 2002, the M.Sc. and Ph.D. degrees in electrical engineering from The University of Texas at Dallas, Richardson, in Dec. 2006 and May 2010, respectively, where she was involved in packet content inspection, repetition-based methodologies and hardware implementation.

She is currently a Research Associate at The University of Texas at Dallas collaborating with the Center for Integrated Circuits and Systems and the Quality of Life Technology Laboratory. Her research interests include high-speed packet processing architectures, and biomedical signal processing and behavior analysis methodologies.

Dr. Faezipour is with the IEEE Women in Engineering Group. 


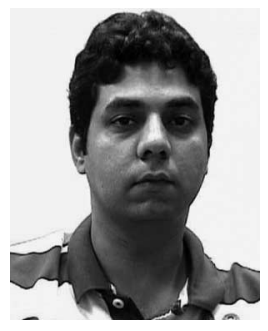

Adnan Saeed received the B.Sc. degree in electrical engineering from the National University of Sciences and Technology, Islamabad, Pakistan, in 2000, the M.Sc. degree in electrical engineering from The University of Texas at Dallas, Richardson, in 2008, where he is involved in the research on sensor nodes for body-area networks. He is currently working toward the Ph.D. degree from the University of Texas at Dallas, where he is also a member of the Center for Integrated Circuits and Systems and Quality of Life Technology Laboratory.

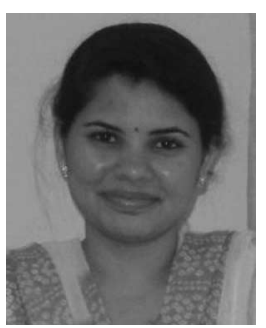

Suma Chandrika Bulusu received the B.E. degree in electronics and communications engineering from the Osmania University, Hyderabad, India, in 2005, the M.S. degree in electrical engineering from the University of Texas at Dallas, Richardson, in May 2010, majoring in signal processing and telecommunications.

Her M.S. thesis focused on ECG transient STepisode detection and machine learning based heart beat classification. She is a Research Assistant at the University of Texas at Dallas in collaboration with the Quality of Life Technology Laboratory. Her research interests include biomedical signal processing, data mining and classification techniques in pattern recognition, and machine learning applied to the biomedical signals.

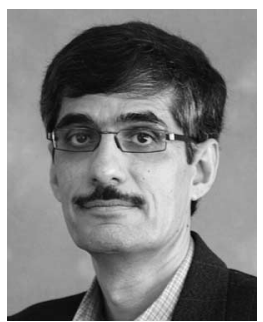

Mehrdad Nourani (S'91-M'94-SM'05) received the B.Sc. and M.Sc. degrees in electrical engineering from the University of Tehran, Tehran, Iran, and the $\mathrm{Ph} . \mathrm{D}$. degree in computer engineering from Case Western Reserve University, Cleveland, $\mathrm{OH}$.

He was with the Department of Electrical and Computer Engineering, University of Tehran, from 1995 to 1998 and the Department of Electrical Engineering and Computer Science, Case Western Reserve University, from 1998 to 1999 . Since August 1999, he has been on the Faculty of the University of Texas at Dallas, where he is currently an Associate Professor of electrical engineering and a member of the Center for Integrated Circuits and Systems. He is a cofounder of the Quality of Life Technology Laboratory in the University of Texas at Dallas, where he is involved in the development of innovative technology and systems that improve people's quality of life. He is the author or coauthor of more than 150 papers in journals and refereed conference proceedings. His current research interests include fault-tolerant architectures, system-on-chip testing, signal integrity modeling and test, application specific architectures for medical applications and high-speed packet processing methodologies and architectures.

Dr. Nourani was a recipient of the Clark Foundation Research Initiation Grant in 2001, the National Science Foundation Career Award in 2002, the Best Paper Award at the 2004 International Conference on Computer Design (ICCD), and Cisco Systems Inc. URP Award in 2004 and 2010, respectively. He is a member of the IEEE Computer Society and the Association for Computing Machinery (ACM) Special Interest Group on Design Automation (SIGDA).

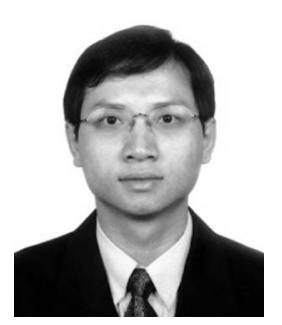

Hlaing Minn (S'99-M'01-SM'07) received the B.E. degree in electronics from the Yangon Institute of Technology, Yangon, Myanmar, in 1995, the M.Eng. degree in telecommunications from the Asian Institute of Technology (AIT), Pathumthani, Thailand, in 1997, and the Ph.D. degree in electrical engineering from the University of Victoria, Victoria, BC, Canada, in 2001

He was with the Telecommunications Program in AIT as a Laboratory Supervisor during 1998. He was a Research Assistant from 1999 to 2001, and a Postdoctoral Research Fellow during 2002 in the Department of Electrical and Computer Engineering at the University of Victoria. He has been with the Erik Jonsson School of Engineering and Computer Science, the University of Texas at Dallas, Richardson, since 2002, and currently is an Associate Professor. His research interests include wireless communications, statistical signal processing, error control, detection, estimation, synchronization, signal design, cross-layer design, cognitive radios, and wireless healthcare applications.

Dr. Minn is an Editor for the IEEE TRANSACTIONS ON COMMUNICATIONS and International Journal of Communications and Networks.

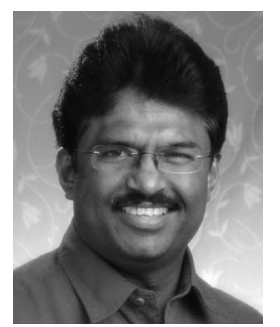

Lakshman Tamil (S'81-M'88-SM'05) received the $\mathrm{Ph} . \mathrm{D}$. degree in electrical engineering and the M.S. degree in mathematics from the University of Rhode Island, in 1989, the M.Tech degree in microwave and optical communication engineering from the Indian Institute of Technology, Kharagpur, India, in 1983, and the B.E. degree in electronics and communication engineering from the Madurai Kamaraj University, Madurai, India, in 1981.

During 2000-2002, he was the CEO and CTO of Yotta Networks Inc., Richardson, TX, a venture funded startup that developed and marketed terabit switching platforms 19971999, he was Section Head of optics and directed research in optical switching, routing, and networks at the North American Alcatel Research Laboratory, Richardson, TX. He was also a consultant for the Naval Research Laboratories Raytheon, Alcatel, Spike Technologies, and Electrospace. He was a Leader in creating both the first Multi-Terabit hybrid optical internet protocol router and a multichannel multipoint distribution service that was a precursor to the Wi-Max. He is currently a Professor of electrical engineering and leads the Quality of Life Technology Laboratory at the University of Texas, Dallas. He is the author or coauthor of more than 100 scholarly research publications in journals, conferences, and edited volumes. He has supervised 12 students for doctoral studies and holds 19 U.S. patents. His research interests include quality of life technologies, radio frequency identification, wireless sensor networks, nanophotonics, and optical transmission, switching and routing. 